\title{
Ventilación de alta frecuencia oscilatoria en pacientes pediátricos
}

\author{
ALEJANDRO DONOSO F.*, PABLO CRUCES R.**, JOSÉ LEÓN B.* \\ MILENA RAMÍREZ A.* y JORGE CAMACHO A.*
}

\section{High-frequency oscillatory ventilation in pediatric patients}

Introduction: High-frequency oscillatory ventilation (HFOV) uses small tidal volumes, often smaller than the anatomic dead space, with high respiratory rates $(>1 \mathrm{~Hz})$. This therapeutic option has become more popular in our country in the last years. Study objectives: To review the clinical experience with HFOV in our Pediatric Intensive Care Unit and to describe the patient's characteristics, HFOV strategies and outcome. Patients: A prospective study included patients less than 16 years old with Acute Respiratory Distress Syndrome (ARDS) refractary to conventional mechanical ventilation (CMV). We compare two periods (1999-2001 and 2002-2004). Measurements and results: Forty-nine patients underwent onto 51 episodes of HFO ventilation, $80 \%$ presented with primary ARDS. Previous conventional ventilation was 47 hours long. The median value of the oxygenation index (OI) at the beginning of HFOV was 24. Mean airway pressure was initially set at $9 \mathrm{cmH}_{2} \mathrm{O}$ above the value in conventional ventilation. All the patients presented a decrease of their $O I$ and ventilation was improved during the first 48 hours of therapy. Median duration on HFOV was 102 hours. The most frequent side effect was transient hypotension (25\%). In the second period we observed a decrease in hemodynamic complications and also an increase in HFO duration. The mortality rate was $33 \%$ (22\% due to pulmonary cause). Conclusion: HFOV is an effective therapy for pediatric respiratory failure refractory to conventional mechanical ventilation.

Key words: High-frequency oscillatory ventilation, Mechanical ventilation, Respiratory failure, Acute respiratory distress syndrome, Pediatric intensive care.

\section{Resumen}

Introducción: La ventilación de alta frecuencia oscilatoria (VAFO) es una modalidad que emplea pequeños volúmenes corrientes, habitualmente menores que el espacio muerto anatómico, con rápidas frecuencias respiratorias (> $1 \mathrm{~Hz}$ ). Esta opción ha sido cada vez más frecuente de disponer en nuestro país en los últimos años. Objetivos: Revisar la experiencia clínica con el uso de VAFO en nuestra UCI y describir las características de los pacientes, estrategia de VAFO empleada y pronóstico. Pacientes: Un estudio prospectivo, no controlado, de series de casos incluyó pacientes menores de 16 años, con Síndrome de Distress Respiratorio Agudo (SDRA), frente a fracaso de Ventilación Mecánica Convencional (VMC). Se compararon dos períodos dentro del estudio (19992001 y 2002-2004). Resultados: 51 episodios de empleo de VAFO, en 49 pacientes cuyo diagnóstico fue SDRA de causa pulmonar en el 80\%. La duración de la VMC previo a la VAFO fue de 47 horas. El índice de oxigenación al iniciarse la VAFO fue 24. La presión media de vía aérea fue fijada en $9 \mathrm{~cm} \mathrm{H}_{2} \mathrm{O}$ sobre el valor en VMC. Se logró una significativa mejoría en la oxigenación y en la ventilación. La duración media de la VAFO fue de 102 horas. El efecto adverso más frecuente fue la hipotensión transitoria (25\%). En el segundo período analizado hubo una disminución de las complicaciones hemodinámicas ( $p<0,05)$, como también un incremento en la duración de la VAFO. Un 33\% de los pacientes fallecieron, un 22\% por causa pulmonar. Conclusión: La VAFO es una terapia eficaz de soporte ventilatorio ante fracaso de la VMC.

Palabras claves: Ventilación de alta frecuencia oscilatoria, Ventilación mecánica, Insuficiencia respiratoria, Síndrome de distress respiratorio agudo, Cuidados críticos pediátricos.

\footnotetext{
* Médico. Área de Cuidados Críticos, Unidad de Gestión Clínica del Niño. Hospital Padre Hurtado.

** Médico. Programa de Medicina Intensiva Infantil. Clínica Alemana. Hospital Padre Hurtado. Universidad del Desarrollo.
} 


\section{Introducción}

Es conocido que la ventilación mecánica es necesaria para asegurar un adecuado intercambio gaseoso en el Síndrome de Distress Respiratorio Agudo (SDRA) y que puede inducir daño pulmonar y contribuir al desarrollo de disfunción multiorgánica. Cambios cíclicos de volumen pulmonar generan sobredistensión y/o colapso de áreas pulmonares, lo que sería un factor determinante para un eventual daño ${ }^{1}$. Es por ello, que las estrategias ventilatorias protectoras buscan el empleo de bajos volúmenes corrientes, generalmente asociado a PEEP elevado e hipercapnia permisiva, perfilándose en forma promisoria en pacientes que padecen dicha patología ${ }^{2,3}$. Pero en ocasiones, ante el fracaso de esta modalidad terapéutica, cada vez más se ha desarrollado en nuestras Unidades de Cuidados Intensivos el uso de la ventilación de alta frecuencia oscilatoria (VAFO).

Así ventilando por medio de la VAFO en una zona de seguridad situada entre los puntos de inflexión superior e inferior de la curva presiónvolumen estática, se evitan los ciclos de colapso pulmonar seguidos de sobredistensión pulmonar. La VAFO cumple teóricamente los ideales de un modo de ventilación eficaz, es decir, pequeños volúmenes corrientes y leves oscilaciones sobre una presión media de vía aérea, en un intento de limitar el riesgo de sobredistensión de las unidades alveolares regionales y volutrauma ${ }^{4}$.

En nuestro país las publicaciones del uso de VAFO en población pediátrica son aún muy escasas $^{5}$, por lo cual parece relevante dar a conocer esta serie con nuestra casuística, complementaria a la previamente comunicada ${ }^{6}$, la más importante publicada hasta la fecha.

\section{Objetivos}

\section{General}

Describir el uso de VAFO en niños menores de 16 años de edad como tratamiento del SDRA luego de fracaso de la ventilación mecánica convencional.

\section{Especificos}

a) Describir las características clínicas de los pacientes con SDRA tratados con VAFO.

b) Describir los parámetros ventilatorios utilizados.

c) Mostrar la eficacia del uso de la VAFO según el protocolo empleado.

d) Describir los efectos adversos asociados a VAFO. e) Analizar curvas de respuesta en los indicadores de oxigenación y ventilación.

f) Determinar relación entre evolución de indicadores de oxigenación y letalidad

g) Comparar los primeros pacientes tratados con VAFO con los más recientes, para identificar curva de aprendizaje.

\section{Pacientes y Método}

Durante un período de 64 meses (mayo de 1999 a agosto de 2004) se reclutaron todos los pacientes menores de 16 años de edad que ingresaron al Área de Cuidados Críticos del Hospital Padre Hurtado con criterios diagnósticos de $\operatorname{SDRA}^{7}$ y que presentaron fracaso al tratamiento empleado con Ventilación Mecánica Convencional (VMC), definido éste como presentar un índice de oxigenación (IO) $>16$ (IO = PMVA $x \mathrm{FiO}_{2} / \mathrm{PaO}_{2}$ x 100) al menos en dos mediciones consecutivas por un período de cuatro horas. No se excluyeron pacientes en shock.

Se midieron las variables demográficas edad, peso, sexo y diagnóstico primario; variables de VMC y VAFO como tiempo de ventilación, parámetros ventilatorios (presión media de vía aérea: PMVA, frecuencia ventilatoria, amplitud de presiones), gases arteriales, $\mathrm{PaO}_{2} / \mathrm{FiO}_{2}$ e IO al ingreso y a las $6,12,24$ y $48 \mathrm{~h}$ y final. Se registraron las complicaciones hemodinámicas (taquicardia, hipotensión) y respiratorias (barotrauma) y la sobrevida o fallecimiento. Se definió hipotensión arterial como una caída > 20\% del valor de la presión arterial sistémica luego del inicio de la VAFO.

La modalidad ventilatoria convencional empleada correspondió a presión control (Evita 2 Dura-Dräger), limitando la presión inspiratoria máxima a no más de $30 \mathrm{~cm} \mathrm{H}_{2} \mathrm{O}$, con una estrategia de hipercapnia permisiva $\left(\mathrm{PaCO}_{2}\right.$ para $\mathrm{pH}$ mayor a 7,1). La $\mathrm{FiO}_{2}$ fue aquella que permitiese una saturación de $\mathrm{O}_{2}$ mayor a 90\%. El PEEP empleado se tituló para el que lograse el mejor IO y/u obtener una $\mathrm{FiO}_{2}<0,6$. Si con lo previo no se lograba una adecuada oxigenación, se empleó decúbito prono. No se ocupó inversión de la relación inspiración/espiración. No se efectuó maniobra de reclutamiento alveolar. Todos los pacientes en $\mathrm{VMC}$ fueron sedoparalizados con midazolam $(0,1-0,4 \mathrm{mg} / \mathrm{kg} / \mathrm{h})$ y fentanilo (1-4 $\mu \mathrm{g} / \mathrm{kg} / \mathrm{h})$ más vecuronio $(0,1-0,4 \mathrm{mg} / \mathrm{kg} / \mathrm{h})$ en infusión continua. Se monitorizaron con saturometría arterial continua (Oxypleth Novametrix, Medical Systems Inc.), capnografía $\left(\mathrm{CO}_{2}\right.$ Sensor SpaceLabs Medical. Redmond, Washington, 
USA) y curvas de mecánica ventilatoria (Navigator Newport Medical Instruments, Newport Beach, California, USA). En el aspecto hemodinámico esta monitorización fue con línea arterial, catéter venoso central y en algunos casos con ecocardiografia doppler o catéter de arteria pulmonar.

Se empleó el ventilador de alta frecuencia oscilatoria SensorMedics 3100A (Yorba Linda, California, USA). Los parámetros iniciales de la VAFO fueron fijados según el siguiente protocolo preestablecido:

- PMVA inicialmente al menos de $5 \mathrm{~cm} \mathrm{H}_{2} \mathrm{O}$ sobre la última en VM convencional, luego con incrementos de $2 \mathrm{~cm} \mathrm{H}_{2} \mathrm{O}$ hasta observarse una mejoría de la oxigenación (saturación de hemoglobina mayor a 90\%) y sin elementos de hiperinsuflación en la radiografía de tórax (diafragma en T8-T9).

- $\mathrm{FiO}_{2}$ inicial de 1, la cual se disminuye paulatinamente hasta 0,6 para obtener una saturación arterial mayor de $90 \%$.

- La presión de amplitud (delta P) fue aquella que causó una adecuada movilidad de la pared torácica, corroborada con valor de $\mathrm{PaCO}_{2}$ en gasometría arterial en la primera hora. Para lograr una disminución de la $\mathrm{PaCO}_{2}$ se incrementó el delta $\mathrm{P}$ en 2 a $5 \mathrm{~cm} \mathrm{H}_{2} \mathrm{O}$ progresivamente y de no obtenerse respuesta se disminuyó la frecuencia respiratoria en 1 a 2 $\mathrm{Hz}$ hasta un mínimo de $3 \mathrm{~Hz}$ de ser necesario y se asoció a desinflar el cuff del tubo endotraqueal en caso de disponer de esta alternativa.

- La frecuencia respiratoria se indicó según el peso del paciente, siendo de $15 \mathrm{~Hz}$ para 500 g a $2 \mathrm{~kg}, 10 \mathrm{~Hz}$ para 4 a $12 \mathrm{~kg}, 8 \mathrm{~Hz}$ para 13 a $20 \mathrm{~kg}, 7 \mathrm{~Hz}$ para 21 a $30 \mathrm{~kg}$ y $5 \mathrm{~Hz}$ para mayores de $30 \mathrm{~kg}$.

- El tiempo inspiratorio se mantuvo en $33 \%$ y el bias flow en $20 \mathrm{~L} / \mathrm{min}$ en todas las oportunidades.

- Se ocupó estrategia de "bajo volumen pulmonar" en casos en que la condición fisiopatológica subyacente fuera de escape aéreo persistente. Ella consistió en reducir la PMVA hasta que la fuga aérea cesara, dando de este modo prioridad a la reducción de la PMVA, aportando una mayor $\mathrm{FiO}_{2}$ durante ese periodo.

- Para desconectar de VAFO se efectuó un descenso progresivo de la PMVA en 1 a 2 $\mathrm{cm} \mathrm{H}_{2} \mathrm{O}$ hasta lograr una presión menor a 20 $\mathrm{cm} \mathrm{H}_{2} \mathrm{O}$, manteniendo una $\mathrm{FiO}_{2}$ menor a 0,60,5 , con saturación mayor a $92 \%$, como tam- bién un descenso del delta $\mathrm{P}$ de 2 a $5 \mathrm{~cm}$ $\mathrm{H}_{2} \mathrm{O}$ para una $\mathrm{PaCO}_{2}$ adecuada según el contexto clínico. Debía observarse también una mejoría radiológica con ausencia de barotrauma, como también una buena tolerancia a la aspiración del TET (mínima desaturación, con rápida recuperación de la oxigenación).

- La permeabilización de la vía aérea, con bolsa y válvula de PEEP en $10 \mathrm{~cm} \mathrm{H}_{2} \mathrm{O}$, siempre se efectuó previo a la conexión a VAFO y luego en las oportunidades que el juicio clínico así lo indicase, a saber, cambio en el status respiratorio (alteración en los gases), secreciones visibles en el tubo endotraqueal (TET), disminución de las vibraciones de la pared toráxica, no dejando pasar nunca más allá de 12 horas.

- Una vez cumplido el protocolo de VAFO, todos los pacientes fueron conectados a VMC (presión control).

Se consideró fracaso del tratamiento cuando no se logró los objetivos terapéuticos previamente señalados.

Se definió letalidad al momento de alta de UCI. Se separaron las causas de fallecimiento en respiratoria o no respiratoria.

Se comparó el período inicial (A) de uso de VAFO con el posterior (B), comprendiendo los años 1999-2001 y 2002-2004 respectivamente.

\section{Análisis Estadístico}

Se efectuaron mediciones de proporciones y medianas para las distintas variables debido al tamaño muestral pequeño. El intervalo de confianza del 95\% (IC95) fue realizado con el método cuadrático de Fleiss calculado en el programa Epi Info 6.04d. El test estadístico fue la prueba de medianas con prueba de $\chi^{2}$ con corrección de Yates, considerando significativo un valor de $\mathrm{p}$ inferior a 0,05 .

\section{Resultados}

\section{Características de la muestra}

Durante el período del estudio se efectuaron 51 tratamientos consecutivos de VAFO en 49 pacientes (2 pacientes con 2 conexiones) obteniéndose un total de 7563 horas de uso de VAFO. Las características demográficas, valores gasométricos, horas de ventilación mecánica y condición de egreso se exponen en las Tablas 1 y 2, separados según el período evaluado. 
La causa más frecuente de SDRA fue pulmonar en 39 pacientes (80\%). De los 10 pacientes con SDRA de causa extrapulmonar, 8 presentaron shock séptico.

En la Tabla 3 se resumen las características demográficas, de ventilación mecánica convencional y oscilatoria, valores gasométricos e indicadores de oxigenación entre los pacientes vivos y muertos. No se observó diferencia estadísticamente significativa en peso, edad, diagnóstico primario, indicadores de oxigenación $\left(\mathrm{PaO}_{2}\right)$ $\mathrm{FiO}_{2}$ e IO) y valor de $\mathrm{PaCO}_{2}$ entre ambos grupos. Solo se observó una diferencia significativa entre el IO final entre ambos grupos. La Tabla 4 muestra los mismos datos pero separados en los periodos A y B.

\section{Ventilación mecánica convencional:}

La mediana de duración de la VMC previa a la VAFO fue de 47 horas (rango de 0-740). Los parámetros al considerar fracaso de VMC fueron $\mathrm{PMVA}$ de $16 \mathrm{cmH}_{2} \mathrm{O}\left(9-28 \mathrm{~cm} \mathrm{H}_{2} \mathrm{O}\right), \mathrm{PaO}_{2}$ / $\mathrm{FiO}_{2}$ de 65 (30 a 174) e IO de 24 (17-75). Los valores gasométricos arteriales mostraron un $\mathrm{pH}$ de 7,2 $(6,9-7,6)$ y una $\mathrm{PaCO}_{2}$ de $64 \mathrm{mmHg}(17-$ $137 \mathrm{mmHg}$ ).

Tabla 1. Datos demográficos, horas de ventilación y pronóstico de pacientes sometidos a ventilación de alta frecuencia oscilatoria (VAFO) en el periodo A, comprendido entre 1999 y 2001

\begin{tabular}{|c|c|c|c|c|c|c|c|c|}
\hline Caso & $\begin{array}{c}\text { Edad } \\
(\mathbf{m})\end{array}$ & $\begin{array}{c}\text { Peso } \\
\text { (kg) }\end{array}$ & Diagnóstico primario & $\begin{array}{c}\mathrm{PaO}_{2} / \\
\mathrm{FiO}_{2}\end{array}$ & IO & $\begin{array}{c}\text { VMC } \\
\text { (h) }\end{array}$ & $\begin{array}{c}\text { VAFO } \\
\text { (h) }\end{array}$ & $\begin{array}{l}\text { Condición } \\
\text { de egreso }\end{array}$ \\
\hline 1 & 3 & 4,8 & BNM Pj + Influenza A & 85 & 20 & 120 & 60 & vivo \\
\hline 2 & 11 & 9 & BNM & 47 & 32 & 33 & 480 & fallecido \\
\hline 3 & 5 & 6 & BNM necrotizante & 80 & 20 & 108 & 504 & fallecido \\
\hline 4 & 2 & 5,4 & Neumonitis varicelatosa & 63 & 24 & 12 & 116 & vivo \\
\hline 5 & 5 & 4,8 & Shock séptico & 45 & 44 & 16 & 80 & fallecido \\
\hline 6 & 8 & 7,5 & BNM & 84 & 17 & 9 & 148 & fallecido \\
\hline 7 & 2 & 3,2 & BNM - DBP- Coqueluche & 154 & 40 & 0 & 237 & fallecido \\
\hline 8 & 3 & 4 & $\mathrm{BNM}$ VRS + ADV - DBP & 35 & 46 & 240 & 40 & vivo \\
\hline $9 *$ & 55 & 15 & Shock Séptico & 66 & 20 & 12 & 43 & fallecido \\
\hline 10 & 37 & 17 & Shock séptico & 61 & 33 & 24 & 92 & vivo \\
\hline 11 & 5 & 4,3 & Coqueluche - BNM & 56 & 30 & 8 & 6 & fallecido \\
\hline 12 & 37 & 17 & Neumonía - Barotrauma & 37 & 43 & 240 & 96 & vivo \\
\hline 13 & 104 & 60 & Shock séptico & 51 & 39 & 6 & 80 & vivo \\
\hline 14 & 1 & 3,5 & BNM Influenza A & 67 & 21 & 31 & 84 & vivo \\
\hline 15 & 83 & 11 & BNM VRS & 43 & 35 & 36 & 80 & vivo \\
\hline 16 & 1 & 8 & BNM parainfluenza & 80 & 21 & 38 & 100 & vivo \\
\hline 17 & 16 & 9,8 & Shock séptico - Hemorragia Pulmonar & 90 & 17 & 0 & 45 & fallecido \\
\hline $18 *$ & 8 & 7,7 & Canal AV Shock Séptico & 63 & 22 & 740 & 110 & vivo \\
\hline 19 & 4 & 4,2 & BNM & 55 & 27 & 696 & 85 & vivo \\
\hline 20 & 1 & 4 & BNM & 79 & 20 & 72 & 96 & vivo \\
\hline 21 & 1 & 4 & Coqueluche - BNM & 75 & 21 & 6 & 20 & fallecido \\
\hline 22 & 16 & 9,5 & BNM & 100 & 17 & 36 & 96 & vivo \\
\hline 23 & 2 & 6 & LLA & 44 & 45 & 17 & 54 & vivo \\
\hline 24 & 6 & 6 & BNM - IC & 61 & 32 & 264 & 360 & fallecido \\
\hline 25 & $15 \mathrm{~h}$ & 4,5 & BNM connatal & 32 & 75 & 14 & 51 & vivo \\
\hline 26 & $3 d$ & 3 & SAM & 33 & 61 & 63 & 93 & vivo \\
\hline 27 & 3 & 2,8 & BNM - DBP & 50 & 65 & 27 & 67 & vivo \\
\hline 28 & 4 & 6 & BNM & 94 & 17 & 8 & 156 & vivo \\
\hline 29 & 67 & 18 & Adenoflegmón - Shock Séptico & 39 & 57 & 228 & 263 & vivo \\
\hline
\end{tabular}

*= Dos episodios de VAFO. IO = Índice de Oxigenación. VMC = Ventilación mecánica convencional. BNM = Bronconeumonía. LLA = Leucemia linfoblástica aguda. $\mathrm{Pj}=$ Pneumocystis jiroveci . VRS = Virus respiratorio sincicial. $\mathrm{ADV}=$ Adenovirus. SAM $=$ Síndrome aspirativo meconial. $\mathrm{DBP}=$ Displasia Broncopulmonar. $\mathrm{IC}=\mathrm{Inmunodeficiencia}$ combinada. $\mathrm{m}=$ meses. $\mathrm{h}=$ horas. $\mathrm{d}=$ días . 
Tabla 2. Datos demográficos, horas de ventilación y pronóstico de pacientes sometidos a ventilación de alta frecuencia oscilatoria (VAFO) en el período B, comprendido entre 2002 y 2004

\begin{tabular}{|c|c|c|c|c|c|c|c|c|}
\hline Caso & $\begin{array}{c}\text { Edad } \\
(\mathrm{m})\end{array}$ & $\begin{array}{c}\text { Peso } \\
(\mathrm{kg})\end{array}$ & Diagnóstico primario & $\begin{array}{c}\mathrm{PaO}_{2} / \\
\mathrm{FiO}_{2}\end{array}$ & IO & $\begin{array}{c}\text { VMC } \\
\text { (h) }\end{array}$ & $\begin{array}{c}\text { VAFO } \\
\text { (h) }\end{array}$ & $\begin{array}{l}\text { Condición } \\
\text { de egreso }\end{array}$ \\
\hline 30 & 1 & 2,7 & BNM VRS & 88 & 17 & 48 & 456 & vivo \\
\hline 31 & $10 \mathrm{~d}$ & 2,8 & $\mathrm{BNM}$ & 30 & 57 & 96 & 658 & fallecido \\
\hline 32 & 7 & 4,4 & BNM Aspirativa & 112 & 17 & 120 & 90 & vivo \\
\hline 33 & 15 & 12 & BNM - Barotrauma & 100 & 18 & 15 & 177 & vivo \\
\hline 34 & 2 & 5 & Coqueluche & 85 & 19 & 240 & 121 & fallecido \\
\hline 35 & 135 & 50 & Hepatitis - Cirrosis & 138 & 18 & 0 & 220 & fallecido \\
\hline 36 & 25 & 12 & BNM & 174 & 17 & 152 & 250 & vivo \\
\hline 37 & 4 & 5 & Shock Séptico & 39 & 64 & 72 & 123 & fallecido \\
\hline 38 & 30 & 15 & Neumonía - Miopatía & 100 & 17 & 36 & 105 & vivo \\
\hline 39 & 2 & 4,1 & Coqueluche - BNM & 80 & 21 & 168 & 106 & fallecido \\
\hline 40 & $15 \mathrm{~d}$ & 3,5 & BNM VRS & 92 & 17 & 72 & 170 & vivo \\
\hline 41 & 10 & 8,5 & BNM VRS & 50 & 36 & 144 & 269 & fallecido \\
\hline 42 & 9 & 10,5 & Neumonía - Shock Séptico & 59 & 42 & 66 & 162 & vivo \\
\hline 43 & $13 \mathrm{~h}$ & 4,2 & BNM connatal & 61 & 40 & 12 & 142 & vivo \\
\hline 44 & 2 & 2,7 & BNM - Neumotórax & 55 & 29 & 180 & 185 & vivo \\
\hline 45 & $12 \mathrm{~h}$ & 3,5 & SAM & 41 & 39 & 6 & 192 & vivo \\
\hline 46 & 74 & 28 & Pleuroneumonía- Neumotórax & 58 & 48 & 12 & 61 & vivo \\
\hline 47 & 11 & 5 & BNM ADV & 74 & 35 & 240 & 156 & vivo \\
\hline 48 & 2 & 2,5 & BNM & 135 & 18 & 84 & 84 & vivo \\
\hline 49 & 12 & 12 & BNM VRS - Neumotórax & 66 & 24 & 72 & 132 & vivo \\
\hline
\end{tabular}

IO = Índice de Oxigenación. VMC = Ventilación mecánica convencional. BNM = Bronconeumonía. VRS = Virus respiratorio sincicial. $\mathrm{ADV}=$ Adenovirus. $\mathrm{SAM}=$ Síndrome aspirativo meconial. $\mathrm{m}=$ meses. $\mathrm{h}=\mathrm{horas} . \mathrm{d}=$ días .

\section{Ventilación de alta frecuencia oscilatoria:}

Los valores de inicio de VAFO fueron PMVA de $26 \mathrm{~cm} \mathrm{H}_{2} \mathrm{O}$ (rango de 17-41 $\mathrm{cm} \mathrm{H}_{2} \mathrm{O}$ ), $\mathrm{FiO}_{2}$ de 1 (0,6-1), delta $\mathrm{P}$ de $50 \mathrm{~cm} \mathrm{H}_{2} \mathrm{O}$ (34-64 $\left.\mathrm{cmH}_{2} \mathrm{O}\right)$ y frecuencia de $10 \mathrm{~Hz}(5-12 \mathrm{~Hz}) . \mathrm{La}$ diferencia de presión media entre la transición de VMC a VAFO fue de $9 \mathrm{~cm}_{2} \mathrm{O}$. La mediana de duración de la VAFO fue de 102 horas con un rango entre 6 y 660 horas.

\section{Variables del intercambio de gases:}

La Figura 1 presenta la evolución temporal de la respuesta de la oxigenación, representado por el IO, previo a VAFO, durante las primeras 48 horas de la terapia y al momento final. En ella se aprecia un incremento estadísticamente significativo del IO entre el momento previo e inmediatamente de iniciada la VAFO. Posteriormente existe un descenso progresivo del IO en el tiempo, analizado tanto en el grupo total como en los vivos y fallecidos por separado, siendo significativa la mejoría del IO a las $6,12,24$ y $48 \mathrm{~h}$ y final comparado con el momento inicial de la terapia oscilatoria $(0$ h), como también se observó una diferencia significativa en el IO final entre el grupo de pacientes vivos y fallecidos.

La Figura 2 muestra evolución en el tiempo de la ventilación alveolar, apreciándose una significativa disminución de los valores de $\mathrm{PaCO}_{2}$ apreciable desde la hora inicial, la que se mantiene en el tiempo.

\section{Complicaciones:}

En 13 de los episodios ventilatorios (25\% con $\mathrm{IC}_{95}=15-40 \%$ ) se consignó la existencia de complicación hemodinámica, describiéndose hipotensión arterial asociada a taquicardia. Hubo barotrauma en 19 de las 51 conexiones, en 11 ya era previo y sólo en 8 durante la VAFO, resultando un $16 \%$ ( $\mathrm{IC}_{95}=7-29 \%$ ) en relación con la conexión de la VAFO. Se destaca que de los once pacientes con barotrauma previo, falleció sólo un paciente; mientras que de los ocho pacientes con barotrauma intra VAFO fallecieron cinco pacientes, sin existir diferencia estadísticamente significativa. 
Tabla 3. Comparación de variables demográficas de ventilación mecánica convencional y de alta frecuencia oscilatoria (VAFO) según condición de egreso de la totalidad de la muestra

\begin{tabular}{|c|c|c|c|c|c|}
\hline Variable & & Vivos & \multicolumn{2}{|c|}{ Fallecidos } & Valor $p$ \\
\hline \multicolumn{6}{|c|}{ Datos demográficos: } \\
\hline Número total & 33 & & 16 & & - \\
\hline Edad (meses) & 4 & $(12 \mathrm{~h}$ a 104$)$ & 5 & $(10 \mathrm{~d}$ a 135$)$ & NS \\
\hline Sexo masculino & 19 & & 12 & & NS \\
\hline Peso (kg) & 5,7 & $(2,5$ a 60$)$ & 6 & $(2,8$ a 50$)$ & NS \\
\hline \multicolumn{6}{|l|}{ VMC: } \\
\hline Duración (h) & 56 & (6 a 740) & 39,5 & (0 a 264) & NS \\
\hline PMVA $\left(\mathrm{cmH}_{2} \mathrm{O}\right)$ & 16 & (9 a 28) & 17 & (13 a 27) & NS \\
\hline $\mathrm{PaO}_{2} / \mathrm{FiO}_{2}$ & 63 & (32 a 174) & 68 & (30 a 198) & NS \\
\hline IO & 27 & (17 a 75) & 22 & (17 a 64) & NS \\
\hline $\mathrm{pH}$ & 7,22 & $(6,91$ a 7,57$)$ & 7,25 & $(6,93$ a 7,62$)$ & NS \\
\hline $\mathrm{PaCO}_{2}(\mathrm{mmHg})$ & 69 & (23 a 137) & 61 & (17 a 114) & NS \\
\hline \multicolumn{6}{|l|}{ VAFO: } \\
\hline Duración (h) & 96 & (51 a 496) & 123 & (6 a 658) & NS \\
\hline IO inicial & 33 & (11 a 93) & 36 & (10 a 59) & NS \\
\hline IO $24 \mathrm{~h}$ & 17 & (8 a 58$)$ & 27 & (9 a 134) & NS \\
\hline IO final & 11 & (3 a 25$)$ & 35 & $(10$ a 146$)$ & $<0,05$ \\
\hline
\end{tabular}

$\mathrm{VMC}=$ Ventilación mecánica convencional. PMVA = Presión media de vía aérea. $\mathrm{IO}=$ Índice de oxigenación. NS = No significativo. $\mathrm{h}=$ horas

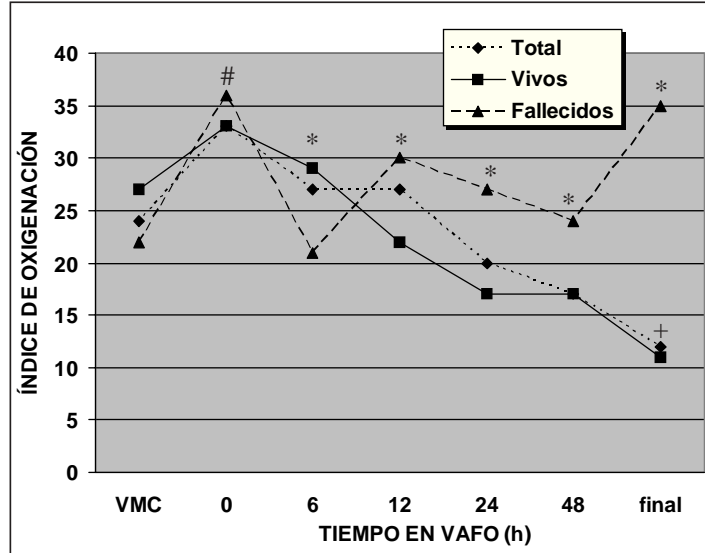

\# indica $\mathrm{p}<0,05$ entre el momento previo e inmediatamente iniciada la VAFO para todos los grupos.

* indica $\mathrm{p}<0,05$ en los distintos períodos de tiempo comprendidos en comparación a hora 0 para todos los grupos.

+ indica $\mathrm{p}<0,05$ en el IO final entre el grupo de los pacientes vivos y fallecidos.

Figura 1. Perfil temporal del índice de oxigenación (IO) en VMC y durante ventilación de alta frecuencia oscilatoria (VAFO) de todos los pacientes, vivos y fallecidos por separado, durante las primeras 48 horas y final.

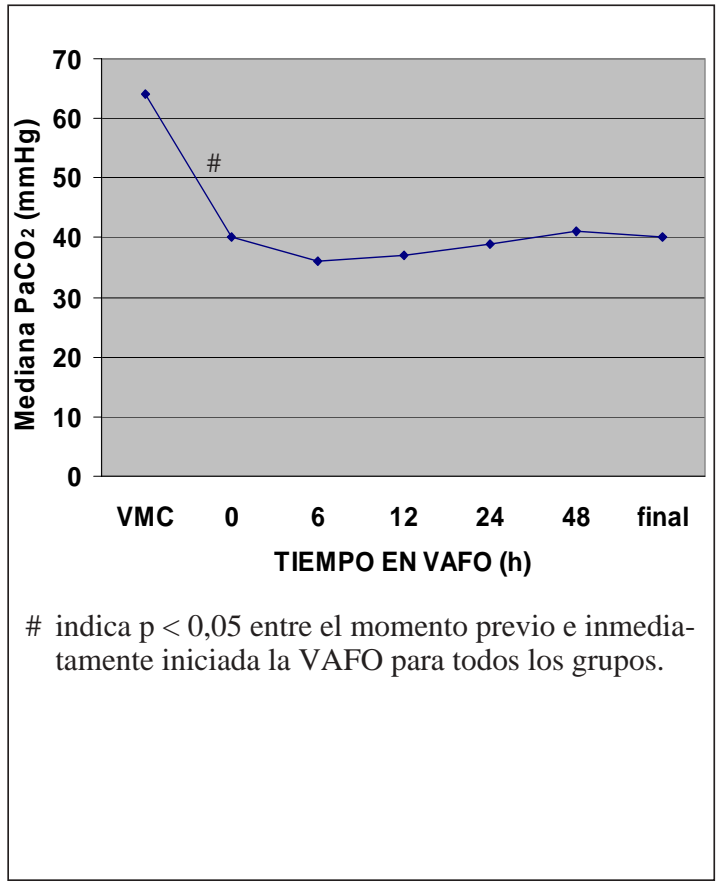

Figura 2. Perfil temporal de la ventilación alveolar en ventilación mecánica convencional y durante ventilación de alta frecuencia oscilatoria (VAFO) de todos los pacientes, durante las primeras 48 horas y final. 
Tabla 4. Comparación de los periodos A (1999-2001) y B (2002-2004) sobre variables demográficas, de ventilación mecánica convencional y de alta frecuencia oscilatoria (VAFO) según condición de egreso

\begin{tabular}{|c|c|c|c|c|c|}
\hline \multirow{2}{*}{$\begin{array}{l}\text { Variable } \\
\text { Período }\end{array}$} & \multicolumn{2}{|c|}{ Vivos } & \multicolumn{2}{|c|}{ Fallecidos } & \multirow[t]{2}{*}{ Valor $\mathrm{p}$} \\
\hline & A & B & $\mathbf{A}$ & B & \\
\hline \multicolumn{6}{|c|}{ Datos demográficos: } \\
\hline Número total & 19 & 14 & 10 & 6 & - \\
\hline Edad (meses) & $\begin{array}{c}3,5 \\
(15 \mathrm{~h} \text { a } 104)\end{array}$ & $\begin{array}{c}8 \\
(12 \mathrm{~h} \text { a } 74)\end{array}$ & $\begin{array}{c}6 \\
(1 \text { a } 55)\end{array}$ & $\begin{array}{c}3 \\
(8 \mathrm{~d} \text { a } 135)\end{array}$ & NS \\
\hline Sexo masculino & 11 & 8 & 9 & 4 & NS \\
\hline Peso (kg) & $\begin{array}{c}6 \\
(2,8 \text { a } 60)\end{array}$ & $\begin{array}{c}4,7 \\
(2,5 \text { a } 28)\end{array}$ & $\begin{array}{c}6 \\
(3,2 \text { a } 15)\end{array}$ & $\begin{array}{c}5 \\
(2,8 \text { a } 50)\end{array}$ & NS \\
\hline \multicolumn{6}{|l|}{ VMC: } \\
\hline Duración (h) & $\begin{array}{c}37 \\
(6 \text { a } 740)\end{array}$ & $\begin{array}{c}69 \\
\text { (6 a } 240)\end{array}$ & $\begin{array}{c}12 \\
(0 \text { a } 264)\end{array}$ & $\begin{array}{c}144 \\
(72 \text { a } 240)\end{array}$ & $<0,05$ \\
\hline PMVA $\left(\mathrm{cmH}_{2} \mathrm{O}\right)$ & $\begin{array}{c}16 \\
(14 \text { a } 24)\end{array}$ & $\begin{array}{c}16 \\
(9 \text { a } 28)\end{array}$ & $\begin{array}{c}16 \\
(13 \text { a } 20)\end{array}$ & $\begin{array}{c}18 \\
(16 \text { a } 27)\end{array}$ & NS \\
\hline $\mathrm{PaO}_{2} / \mathrm{FiO}_{2}$ & $\begin{array}{c}58 \\
(32 \text { a } 100)\end{array}$ & $\begin{array}{c}81 \\
(41 \text { a } 174)\end{array}$ & $\begin{array}{c}68 \\
(45 \text { a } 231)\end{array}$ & $\begin{array}{c}65 \\
(30 \text { a 138) }\end{array}$ & NS \\
\hline IO & $\begin{array}{c}31 \\
(16 \text { a } 75)\end{array}$ & $\begin{array}{c}20 \\
(8 \mathrm{a} 48)\end{array}$ & $\begin{array}{c}22 \\
(6 \mathrm{a} 44)\end{array}$ & $\begin{array}{c}29 \\
(18 \text { a } 64)\end{array}$ & NS \\
\hline $\mathrm{pH}$ & $\begin{array}{c}7,22 \\
(6,99 \text { a } 7,57)\end{array}$ & $\begin{array}{c}7,21 \\
(6,91 \text { a } 7,55)\end{array}$ & $\begin{array}{c}7,21 \\
(6,93 \text { a } 7,51)\end{array}$ & $\begin{array}{c}7,34 \\
(7,18 \text { a } 7,62)\end{array}$ & NS \\
\hline $\mathrm{PaCO}_{2}(\mathrm{mmHg})$ & $\begin{array}{c}72 \\
(25 \text { a } 116)\end{array}$ & $\begin{array}{c}63 \\
(23 \mathrm{a} 137)\end{array}$ & $\begin{array}{c}61 \\
(17 \text { a } 114)\end{array}$ & $\begin{array}{c}52 \\
(28 \text { a } 77)\end{array}$ & NS \\
\hline VAFO: & & & & & \\
\hline Duración (h) & $\begin{array}{c}92 \\
(51 \text { a } 263)\end{array}$ & $\begin{array}{c}149 \\
(61 \text { a } 456)\end{array}$ & $\begin{array}{c}80 \\
(6 \text { a } 504)\end{array}$ & $\begin{array}{c}172 \\
(106 \text { a } 658)\end{array}$ & $<0,05$ \\
\hline IO inicial & $\begin{array}{c}41 \\
(14 \text { a } 93)\end{array}$ & $\begin{array}{c}29 \\
(11 \text { a } 52)\end{array}$ & $\begin{array}{c}37 \\
(10 \text { a } 59)\end{array}$ & $\begin{array}{c}29 \\
(15 \text { a } 47)\end{array}$ & NS \\
\hline IO $24 \mathrm{~h}$ & $\begin{array}{c}20 \\
(8 \text { a } 58)\end{array}$ & $\begin{array}{c}16 \\
(8 \text { a } 28)\end{array}$ & $\begin{array}{c}24 \\
(9 \mathrm{a} 68)\end{array}$ & $\begin{array}{c}27 \\
(15 \text { a } 134)\end{array}$ & NS \\
\hline IO final & $\begin{array}{c}11 \\
(6 \text { a } 25)\end{array}$ & $\begin{array}{c}9 \\
(3 \text { a } 18)\end{array}$ & $\begin{array}{c}31 \\
(10 \text { a } 146)\end{array}$ & $\begin{array}{c}44 \\
(26 \text { a } 146)\end{array}$ & $<0,05$ \\
\hline
\end{tabular}

VMC $=$ Ventilación mecánica convencional. PMVA = Presión media de vía aérea. $\mathrm{IO}=$ Índice de oxigenación. $\mathrm{NS}=$ No significativo. $\mathrm{h}=$ horas 


\section{Condición de egreso:}

La sobrevida fue de $33 / 49$ pacientes $(67 \%$ con $\left.\mathrm{IC}_{95}=52-80 \%\right)$. En 11 de los 16 pacientes fallecidos, la muerte se atribuyó directamente a una causa pulmonar, dando así una letalidad específica de $22 \%$ (con $\mathrm{IC}_{95}=12-37 \%$ ) para la muestra total. Dentro de los 11 pacientes fallecidos por causa pulmonar, 10 obedecieron a SDRA con hipoxemia refractaria, dos de estos en el contexto de Falla Orgánica Múltiple. El otro paciente falleció por enfisema intersticial difuso originado por déficit de proteína $\mathrm{B}$ del surfactante.

\section{Análisis según periodo:}

En el período A hubo una mortalidad de 10/31 (32\%), a su vez que en el período B está fue de $6 / 20(30 \%)$. Con respecto a la existencia de complicaciones, estas fueron para el período A: hemodinámicas 13/31 (42\%), barotrauma 7/31 (23\%) y en el período B fueron de $0 / 20(0 \%)$ y $4 / 20$ (20\%) respectivamente, con $\mathrm{p}<0,05$ para las complicaciones hemodinámicas. La duración de la VAFO fue en el período A de 89 horas y en el período B de 149 horas ( $p<0,05)$, no siendo necesario reconectar pacientes a VAFO en segundo período.

\section{Discusión}

En la última década hemos sido testigos de un notable cambio en los objetivos de la estrategia ventilatoria para el paciente con SDRA; en un inicio se buscaba la mantención de un adecuado intercambio gaseoso (con valores fisiológicos en los gases arteriales) mediante el uso de la ventilación mecánica ${ }^{8,9}$. En 1993 la Conferencia de Consenso, destacó el concepto que el uso de la ventilación mecánica puede causar o agravar el daño pulmonar observado y nos señala los riesgos derivados de la sobredistensión pulmonar, como asímismo el considerar el uso de PEEP ${ }^{10}$. Recientemente en el año 2000, mediante el empleo de una estrategia ventilatoria de protección pulmonar, se observa un impacto en la mortalidad de estos pacientes ${ }^{2}$. Actualmente se continúa en la búsqueda de diversas modalidades con el objetivo de limitar el daño pulmonar asociado a ventilación mecánica (DPAVM). Entre los mecanismos causantes de este podemos identificar la toxicidad por oxígeno ${ }^{11}$, volutrau$\mathrm{ma}^{12-14}$ y atelectrauma ${ }^{15}$, los cuales pueden originar la liberación de citoquinas proinflamatorias (biotrauma), llevando finalmente a la Falla Orgánica Múltiple, la principal causa de muerte en estos pacientes ${ }^{16,17}$. Así la VAFO es una opción ventilatoria con razonables ventajas para ser empleado en este contexto clínico, en la búsqueda de aminorar el DPAVM y a su vez el permitir una adecuado intercambio de gases.

Dependiendo del centro y de la familiaridad del equipo tratante de UCI para el uso de la VAFO, se observan diferencias para el criterio de inicio de la VAFO (falla de ventilación mecánica convencional), de todas formas estas se basan habitualmente en las presiones empleadas, niveles de oxígeno requerido en VMC, respuesta al uso de hipercapnia permisiva y la progresión de la enfermedad. El índice de oxigenación es un indicador de la gravedad de la falla respiratoria que toma en cuenta el costo de presión media de la vía aérea para oxigenar. El valor de corte en 16 para el IO en nuestra casuística es arbitrario, pero está en un rango semejante al presentado por otras importantes series internacionales descritas en la última década ${ }^{18-21}$. Del mismo modo el valor observado en nuestra serie para la $\mathrm{PaO}_{2} / \mathrm{FiO}_{2}$ e IO en la transición desde la VMC y los valores iniciales del IO, son similares a los comunicados por Arnold et al en una importante serie pediátrica multicéntrica ${ }^{22}$.

Así entonces de la serie clínica aquí presentada, debemos enfatizar el concepto derivado de nuestra experiencia, con respecto a que el paciente en quien la VMC no esté logrando los objetivos buscados, basados siempre en un juicio clínico adecuado y en consideración a la progresión del costo de la oxigenación (IO) durante la evolución del SDRA, pase a ser candidato de recibir esta modalidad ventilatoria. Queda pendiente consensuar el valor más adecuado de este índice para el cambio a VAFO.

Observamos que el incremento de la PMVA requerido inicialmente en esta estrategia de apertura pulmonar, mediante el reclutamiento, para lograr una adecuada oxigenación en VAFO, fueron $9 \mathrm{~cm} \mathrm{H}_{2} \mathrm{O}$ sobre los empleados en la ventilación mecánica convencional. Esto avala experiencias de otras series de ocupar valores de PMVA en promedio 6-8 $\mathrm{cm} \mathrm{H}_{2} \mathrm{O}$ sobre el valor previo $^{22}$, cifras bastante superiores a las recomendaciones originales. El fenómeno del reclutamiento alveolar y la mantención de un adecuado volumen pulmonar son de particular importancia en la VAFO, mediante el ajuste de una PMVA sobre la presión de apertura del pulmón $^{23-25}$. Existe concordancia en la literatura, que la omisión de tal maniobra de reclutamiento en los primeros trabajos clínicos de VAFO (especialmente en neonatos) puede en parte dar cuenta de la falta de beneficios observados ${ }^{26}$. 
Hemos sido testigos como en el último tiempo se ha extendido el uso de la VAFO en la población pediátrica más allá del período neonatal $^{22}$ y con efectividad no limitada exclusivamente por el peso del paciente ${ }^{27}$. Así se debe privilegiar entonces el contexto fisiopatológico subyacente más que una limitación a priori originada por el peso.

En concomitancia con la gran mayoría de los estudios publicados las complicaciones hemodinámicas observadas (hipotensión transitoria) fueron escasas y sin repercusión clínica de relevancia y subsanada con aporte de volumen como ha sido descrito en diversos estudios previos ${ }^{28-31}$. Sabemos que la interferencia de la VAFO en la función miocárdica es menor que con VMC a similar valor de $\mathrm{PMVA}^{32}$. Además la pronta y mantenida mejoría observada tanto en la $\mathrm{PaCO}_{2}$ y $\mathrm{PaO}_{2}$ como en el estado ácido-base probablemente se traduce en claros efectos positivos en la situación hemodinámica del niño, permitiendo habitualmente una rápida disminución del apoyo vasoactivo e/o inotrópico requerido.

La mortalidad observada de un tercio de los pacientes ( $22 \%$ causa pulmonar), se asemeja a lo observado en las publicaciones extranjeras ${ }^{22}$. Aunque debemos recordar la distinta población analizada, ya que en nuestra casuística no hubo un grupo importante de pacientes inmunocomprometidos o con cardiopatías complejas cianóticas, las que obviamente constituyen grupos con mayor riesgo de mortalidad ${ }^{22}$. De todas maneras el uso de la VAFO en nuestra experiencia, tiene la característica de ser en una muestra representativa de la realidad pediátrica nacional, con el franco predominio del SDRA de causa pulmonar infecciosa.

Es interesante de comentar derivado del análisis de los dos períodos, la franca disminución de las complicaciones hemodinámicas observadas, lo que probablemente manifieste el haberse habituado el equipo tratante a que estos pacientes deben ser enérgicamente reanimados en búsqueda de una optimización de la precarga, medida generalmente eficaz para evitar el desarrollo de la hipotensión arterial o caídas del débito cardíaco. El otro aspecto a enfatizar es la diferencia en la duración de la VAFO en estos dos períodos, con una mayor estadía en VAFO en el último, esto muy probablemente refleje el concepto de ver actualmente a esta modalidad ventilatoria como una opción de tratamiento, más que sólo como un "rescate de pacientes graves". Es así como en la gran mayoría de nuestros pacientes se tenía una conducta acorde con permitir la estabilización por más de 48 horas, una vez logrados los objetivos gasométricos y radiológicos, antes de convertir a ventilación mecánica convencional

Finalmente, queda por definir en el futuro, entre otros aspectos del uso de VAFO, su real papel en una mejor protección pulmonar al compararlo con estrategias de ventilación mecánica de bajo volumen corriente, la adecuada forma de evaluar en forma no invasiva el volumen pulmonar alcanzado para la correcta titulación de la PMVA ${ }^{33}$, la eficacia al asociarlo a otras terapias como óxido nítrico inhalado ${ }^{19}$, surfactante exógeno ${ }^{34}$, ventilación líquida ${ }^{35} \mathrm{o}$ heliox $^{36}$.

\section{Bibliografía}

1.- DREYFUSS D, SAUMON G: Ventilator-induced lung injury: Lessons from experimental studies. Am J Respir Crit Care Med 1998; 157: 294-323.

2.- Ventilation with lower tidal volumes as compared with traditional tidal volumes for acute lung injury and the acute respiratory distress syndrome. The Acute Respiratory Distress Syndrome Network. N Engl J Med 2000; 342: 1301-8.

3.- AMATO M B, BARBAS C S, MEDEIROS D M, MAGALDI R B, SCHETTINO G P, LORENZI-FILHO G, et al. Effect of a protective - ventilation strategy on mortality in the acute respiratory distress syndrome. N Engl J Med 1998; 338: 347-54.

4.- SINGH J M, STEWART T E. High-frequency mechanical ventilation principles and practices in the era of lung-protective ventilation strategies. Respir Care Clin N Am 2002; 8: 247-60.

5.- RODRÍGUEZ J I, RONCO R, CASTILLO A, GUZMÁN G, VALENZUELA A, PERRET C, et al. Ventilación oscilatoria de alta frecuencia en niños con síndrome de dificultad respiratoria "del adulto". Rev Chil Pediatr 1998; 69: 142-7.

6.- DONOSO A, LEÓN J, ROJAS G, VALVERDE C, ESCOBAR M, RAMIREZ M, et al. Uso de ventilación de alta frecuencia oscilatoria en pacientes pediátricos. Rev Chil Pediatr 2002; 73: 461-70.

7.- BERNARD G R, ARTIGAS A, BRIGHAM K L, CARLET J, FALKE K, HUDSON L, et al. Report of the American-European consensus conference on ARDS: definitions, mechanisms, relevant outcomes and clinical trial coordination. The Consensus Committee. Intensive Care Med 1994; 20: 225-32.

8.- TOBIN MJ. Mechanical ventilation. N Engl J Med 1994; 330: 1056-61.

9.- KOLLEF M H, SCHUSTER D P. The acute respiratory distress syndrome. N Engl J Med 1995; 332: 27-37.

10.- SLUTSKY A S. Mechanical ventilation. American College of Chest Physicians' Consensus Conference. Chest 1993; 104: 1833-59.

11.- BRYAN C L, JENKINSON S G. Oxygen toxicity. Clin Chest Med 1988; 9: 141-52.

12.- DREYFUSS D, SOLER P, BASSET G, SAUMON G. High inflation pressures pulmonary edema. Respective effects of high airway pressure, high tidal volume, and positive end - expiratory pressure. Am Rev Respir Dis 1988; 137: 1159-64. 
13.- CARLTON D P, CUMMINGS J J, SCHEERER R G, POULAIN F R, BLAND R D. Lung overexpansion increases pulmonary microvascular protein permeability in young lambs. J Appl Physiol 1990; 69: 577 83.

14.- HERNÁNDEZ L A, PEEVY K J, MOISE A A, PARKER J C. Chest wall restriction limits high airway pressure-induced lung injury in young rabbits. J Appl Physiol 1989; 66: 2364-8.

15.- MUSCEDERE J G, MULLEN J B, GAN K, SLUTSKY A S. Tidal ventilation at low airway pressures can augment lung injury. Am J Respir Crit Care Med 1994; 149: 1327-34.

16.- SLUTSKY A S, TREMBLAY L N. Multiple system organ failure - Is mechanical ventilation a contributing factor? Am J Respir Crit Care Med 1998; 157: 17215.

17.- TREMBLAY L N, SLUTSKY A S. Ventilator - induced injury: from barotrauma to biotrauma. Proc Assoc Am Physicians 1998; 110: 482-8.

18.- ARNOLD J H, HANSON J H, TORO-FIGUERO L O, GUTIERREZ J, BERENS R J, ANGLIN D L. Prospective randomized comparison of high frequency oscillatory ventilation and conventional mechanical ventilation in pediatric respiratory failure. Crit Care Med 1994; 22: 1530-9.

19.- DOBYNS E L, ANAS N G, FORTENBERRY J D, DESHPANDE J, CORNFIELD D N, TASKER R C, et al. Interactive effects of high-frequency oscillatory ventilation and inhaled nitric oxide in acute hypoxemic respiratory failure in pediatrics. Crit Care Med. 2002; 30: 2425-9.

20.- SLEE-WIJFFELS F Y, VAN DER VAART K R, TWISK J W, MARKHORST D G, PLOTZ F B. Highfrequency oscillatory ventilation in children: a singlecenter experience of 53 cases. Crit Care 2005; 9 (3): R274-9.

21.- GRACIANO A L, FREID E B. High-frequency oscillatory ventilation in infants and children. Curr Opin Anaesthesiol 2002; 15 (2): 161-6.

22.- ARNOLD J H, ANAS N G, LUCKETT P, CHEIFETZ I M, REYES G, NEWTH C J, et al. High-frequency oscillatory ventilation in pediatric respiratory failure: A multicenter experience. Crit Care Med 2000; 28: 3913-9.

23.- FERGUSON N D, KACMAREK R M, MEHTA S, GRANTON J T, LAPINSKY S E, MACDONALD R, et al. Safety and efficacy of high frequency oscillatory ventilation (HFOV) and recruitment maneuvers (RMs) in early severe ARDS. Am J Respir Crit Care Med 2001; 163: A 767.
24.- SUZUKI H, PAPAZOGLOU K, BRYAN A C. Relationship between $\mathrm{PaO}_{2}$ and lung volume during high frequency oscillatory ventilation. Acta Paediatr Jpn 1992; 34: 494-500.

25.- FERGUSON N D, STEWART T E. New therapies for adults with acute lung injury. High-frequency oscillatory ventilation. Crit Care Clin 2002; 18: 91-106.

26.- BRYAN AC, FROESE AB. Reflections on the HIFI trial. Pediatrics 1991; 87: 565-7.

27.- SARNAIK AP, HEIDEMANN SM. Efficacy of highfrequency ventilation for severe hypoxemic respiratory failure in children greater than 30 kilograms. Critical Care Med 1998; 26: 119A.

28.- ARNOLD J H, TRUOG R D, THOMPSON J E, FACKLER J C. High-frequency oscillatory ventilation in pediatric respiratory failure. Crit Care Med 1993; 21: $272-8$.

29.- GUTIÉRREZ J N, LEVIN D L, TORO-FIGUEROA L O. Hemodynamic effects of high-frequency oscillatory ventilation in severe pediatric respiratory failure. Intensive Care Med 1995; 21: 505-10.

30.- MEHTA S, GRANTON J, MACDONALD R J, BOWMAN D, MATTE - MARTYN A, BACHMAN T, et al. High-frequency oscillatory ventilation in adults. The Toronto experience. Chest 2004; 126: 518-27.

31.- GULLBERG N, WINBERG P,SELLDEN H. Changes in mean airway pressure during HFOV influences cardiac output in neonates and infants. Acta Anaesthesiol Scand 2004; 48: 218-23.

32.- TRAVERSE J H, KORVENRANTA H, ADAMS E M, GOLDTHWAIT D A, CARLO W A. Cardiovascular effects of high-frequency oscillatory and jet ventilation. Chest 1989; 96: 1400-4.

33.- GOTHBERG $S$, PARKER T A, GRIEBEL J, ABMAN $S$ $\mathrm{H}$, KINSELLA $J$ P. Changes in thoracic volume in term and preterm lambs measured with inductive plethysmography. Pediatr Res 1999; 45: 303A.

34.- FROESE A B, MCCULLOCH P R, SUGIURA M, VACLAVIK S, POSSMAYER F, MOLLER F. Optimizing alveolar expansion prolongs the effectiveness of exogenous surfactant therapy in the adult rabbit. Am Rev Respir Dis 1993; 148: 569-77.

35.- DOCTOR A, MAZZONI M C, DELBAZO U, DICANZIO J, ARNOLD J H. High-frequency oscillatory ventilation of the perfluorocarbon -filled lung: Preliminary results in an animal model of acute lung injury. Crit Care Med 1999; 27: 2500-7.

36.- WINTERS J W, WILLLING M A, SANFILIPPO D. Heliox improves ventilation during high-frequency oscillatory ventilation in pediatric patients. Pediatr Crit Care Med 2000; 1: 33-37.

\footnotetext{
Correspondencia a:

Dr. Alejandro Donoso F.

E-mail: adonoso@hurtadohosp.cl

Área de Cuidados Críticos

Unidad de Gestión Clínica del Niño

Hospital Padre Hurtado

Santiago. Chile
} 